\title{
Vacuum energy and extension to Machian space-time physics by linkage of gravitons (today) with gravitinos (EW era) as answer to Volovik's "Vacuum Energy, myths and reality" document questions
}

\author{
Andrew Beckwith \\ Department of Physics, Chongqing University \\ E-mail address: \\ abeckwith@uh.edu
}

To cite this article:

Andrew Beckwith. Vacuum Energy and Extension to Machian Space-Time Physics by Linkage of Gravitons (Today) with Gravitinos (Ew Era) as Answer to Volovik'S Vacuum Energy, Myths and Reality Document Questions, American Journal of Modern Physics. Vol. 2, No. 3, 2013, pp. 148-154. doi: 10.11648/j.ajmp.20130203.19

\begin{abstract}
We summarize first the highlights of the paper by Volovik, 2006 as to Vacuum energy and afterwards state that the non applicabillty of "Fjortoft theorem" for defining necessary conditions for instability solves certain problems raised by Volovik. The Myths and Realitites of Vacuum energies as stated by Volovik in particular state that there can be no (local?) perturbations of the quantum vacuum leading to a nonzero vacuum energy. Our paper applies the non applicability of "Fjortoft theorem" as another mechanism which could lead to a nonzero vacuum energy. We apply this theorem to what is called by Padmanabhan a thermodynamic potential which could show inititial conditions implying (structual) instability if conditions for the applications of "Fjortoft's theorem" hold. In our case, there is no instability, so a different mechanism exists.for constructing vacuum energy. We appeal to Machians physics to account for the behavior of massive Gravitons with DE, in sync with extending answers to Volovick's questions and identifying vacuum energy with DE. Then use Branes-Anti branes to create DE. Key point also is in the uniformity of Planck's constant in cosmology, too as to preserve consistency of physical evolution.
\end{abstract}

Keywords: Casmir Effect, Fjortoft Theorem, Thermodynamic Potential, Matter Creation, Higgs Boson, Vacuum Energy. Gravitons, Gravitinos, Mach's Theorem

\section{Introduction: Summary of the} Cosmological Problem, Volovik's Insights and Addendium Added to Get about an (Almost) Zero Value for the Vacuum Energy. Note, Volovik Equates Vacuum Energy with DE (Dark Energy)

To give a starting point we will review a number of the highlights of Volovik's 2006 paper as to Vaccuum energy [1] where in the end Volovik states that perhaps only by application of the Casmir effect, as aexample could Volovik possibly get away fromVolovik's main conclusion. That the natural value of the vacuum energy is zero. Note also that Volovik quotes Einstein that the cosmological constant must be added to Einstein's equations if the density of matter in the universe is non zero in average. So then, quoting Einstein,"The cosmological constant is set equal to zero if matter is so inhomogeneous that its average over big volumes V tends to zero" [2]. When Einstein wrote his statement, he did not know of DM (dark matter) or DE (dark energy) or of the distribution of galaxies in a near fractal pattern, arguing for a high degree of non homogenity, which argues in favor of a near zero cosmological constant, but this must be counterbalanced by how strikingly homogeneous early matter states in the initial big bang really were. Even more unusual is the work by several authors in [3] [4] where Durrer and other authors used a brane world version of the Casmir effect, on a largescale to duplicate conditions for vacuum fluctuations leading to non zero contributions to matter-energy creation. Note that Volovik [1] in his discussion of the Casmir effect asks "Why the zero point energy in the space between the plates (for a Casmir 
effect device) gravitates and not the zero-point energy outside the plates?" Volovik goes on to state that the answer is obvious in that "the local perturbation of the vacuum (by an atom or by plates) does not change the pressure at infinity, and thus the cosmological constant is not perturbed by local perturbations". What this paper does, is to incorporate this insight, as to use the non applicability of the Fjortoft theorem in the initial beginning of the big bang in order to argue in favor of,(after using an early universe thermodynamic potential described by T. Padamanabhan [5] which is stable), to use giant Casmir Plates as to get about the local perturbation effect, which Volovik states dooms the cosmological constant to be almost or exactly zero. We use infinite Casmir plates rerepresented by a brane and anti brane to get about the impossibility of local perturbing effects creating variations in vacuum energy far away from a local space-time geometry. As Volovik [1] identifies vacuum energy with Dark energy at the end of his manuscript, we modify work by Durrer [3],[4] to obtain production of Dark energy via giant Brane-Anti Brane ' Casmir plates'. This will also be a way to reconcile the striking homogenity of the early universe, with its marked present day irregularity.It is worth noting in passing that Volovik assigns DE (dark energy) as the mechanism for the cosmological constant [1]. It is thereby fitting to investigate another mechanism for graviton creation [3],[4] as presented by Durrer et.al as a way to induce massive Graviton creation which Beckwith [6] wrote up as a way to connect gravitons with the re acceleration of the universe problem. The question remains, then, how one can justify a nonzero DE/vacuum energy in the beginning under highly homogeneous conditions, whereas when referencing [2] the vacuum energy tends to zero as spatial inhomogenity of matter dominates.

\section{Organization of the Paper}

The document begins with a statement of Fjortoft theorem [7]. From there, the thermodynamic potential brought up by Thanu Panmanadan [5] is alluded to, and also the failure of the application of Fjortoft theorem to the potential described by [5] by Panmanadan is brought up. I.e. what [5] describes as a complete description of the Early Universe governing thermodynamics. After establishing this, the author assumes that the case is made for a nonzero initial Vacuum energy which is presented in several stages.

a. According to [2] the cosmological constant tends to zero (very small value) if the concentration of matter energy is highly NON uniform (the present day condition). Volovik furthermore states that even in the case of false to true vacuum phase transitions that there is a restoration of a 'condensed matter' type system to effective zero values of vacuum energy. So false to true vacuum phase transitions in themselves do not solve the nonzero Vacuum energy problem [1]. See Volovik for extensive discussions.

b. Initial conditions were very uniform. These are not conditions for a near zero cosmological constant, if Einstein is to be believed [2]. c. Fjortofts theorem makes the case there was no initial instability, i.e. no local space time fluctuations. Therefore, we should not view the universe as a closed system. I.e. some physical mechanism was present to inject matterenergy to create a vacuum energy. As a large value. This statement that the universe is not a closed thermodynamic system is established via use of [5] for a governing thermodynamic potential for the early universe. pp 4-6

d. The Casmir plate analogy, locally, which Volovik cites [2] as insufficient for creating pressure variations on a large / infinite scale was used by Durrer [3], [4] using brane worlds, in the manner similar to Steinhardt and Turok's Ekpyrotic universe [8], [9]. We apply this with tweaking to create vacuum energy, via massive gravitons. This is highly contentious, but a way to come up with a consistent candidate as to DE. Those who dispute this, as to identifying gravitons with DE are welcome to review [6]. pp 6-9

e. Final part, is to appeal to Mach's principle, as a linkage between the electro weak era (gravitinos) and today (gravitons) to preserve the uniformity of Planck's constant to the present era, in the mist of almost unimaginable variation of space time physics from initial conditions to the present. Keeping the uniformity of space - time physics parameters is essential if we wish to have the same physical laws in cosmological evolution to today. pp 9-11

f. Data analysis formalism for $h_{i j}$ metric perturbations as to use of massive gravitons is the finalpart of this paper. pp 11-12

g. Conclusion. i.e. summary of the main results. pp. 1213

\section{Statement of Fjortoft's Theorem. as Used to Start this Paper}

From [7] we have that the theorem to be considered should be written up as follows, namely, look at

Fjortoft theorem:

A necessary condition for instability is that if $z_{*}$ is a point in spacetime for which $\frac{d^{2} U}{d z^{2}}=0$ for any given potential $U$, then there must be some value $z_{0}$ in the range $z_{1}<z_{0}<z_{2}$ such that

$$
\left.\frac{d^{2} U}{d z^{2}}\right|_{z_{0}} \cdot\left[U\left(z_{0}\right)-U\left(z_{*}\right)\right]<0
$$

For the proof, see [1] and also consider that the main discussion is to find instability in a physical system which will be described by a given potential $U$. Next, we will construct in the boundary of the EW era, a way to come up with an optimal description for $U$ 


\section{Constructing an Appropriate Potential for Using Fjortoft Theorem in Cosmology for the Early Universe Cannot Be Done. We Show Why}

To do this, we will look at Padamanabhan [5] and his construction of (in Dice 2010) of thermodynamic potentials he used to have another construction of the Einstein GR equations. To start, Padamanabhan [5] wrote

If $P_{c d}^{a b}$ is a so called Lovelock entropy tensor, and $T_{a b}$ a stress energy tensor

$$
\begin{aligned}
& U\left(\eta^{a}\right)=-4 \cdot P_{a b}^{c d} \nabla_{c} \eta^{a} \nabla_{d} \eta^{b}+T_{a b} \eta^{a} \eta^{b}+\lambda(x) g_{a b} \eta^{a} \eta^{b} \\
& =U_{\text {gravity }}\left(\eta^{a}\right)+U_{\text {matter }}\left(\eta^{a}\right)+\lambda(x) g_{a b} \eta^{a} \eta^{b} \\
& \Leftrightarrow U_{\text {matter }}\left(\eta^{a}\right)=T_{a b} \eta^{a} \eta^{b} ; U_{\text {gravity }}\left(\eta^{a}\right)=-4 \cdot P_{a b}^{c d} \nabla_{c} \eta^{a} \nabla_{d} \eta^{b}
\end{aligned}
$$

We now will look at

$$
\begin{aligned}
& U_{\text {matter }}\left(\eta^{a}\right)=T_{a b} \eta^{a} \eta^{b} ; \\
& U_{\text {gravity }}\left(\eta^{a}\right)=-4 \cdot P_{a b}^{c d} \nabla_{c} \eta^{a} \nabla_{d} \eta^{b}
\end{aligned}
$$

So happens that in terms of looking at the partial derivative of the top (2) equation, we are looking at

$$
\frac{\partial^{2} U}{\partial\left(\eta^{a}\right)^{2}}=T_{a a}+\lambda(x) g_{a a}
$$

Thus, we then will be looking at if there is a specified $\eta_{*}^{a}$ for which the following holds.

$$
\begin{aligned}
& {\left[\frac{\partial^{2} U}{\partial\left(\eta^{a}\right)^{2}}=T_{a a}+\lambda(x) g_{a a}\right]_{\eta_{0}^{a}}} \\
& {\left[\begin{array}{l}
-4 \cdot P_{a b}^{c d}\left(\nabla_{c} \eta_{0}^{a} \nabla_{d} \eta_{0}^{b}-\nabla_{c} \eta_{*}^{a} \nabla_{d} \eta_{*}^{b}\right)+ \\
T_{a b} \cdot\left[\eta_{0}^{a} \eta_{0}^{b}-\eta_{*}^{a} \eta_{*}^{b}\right]+\lambda(x) g_{a b} \cdot\left[\eta_{0}^{a} \eta_{0}^{b}-\eta_{*}^{a} \eta_{*}^{b}\right]
\end{array}\right]}
\end{aligned}
$$

What this is saying is that there is no unique point, using this $\eta_{*}^{a}$ for which (5) holds. Therefore, we say there is no official point of instability of $\eta_{*}^{a}$ due to (4). The Lagrangian structure of what can be built up by the potentials given in (4) with respect to $\eta_{*}^{a}$ mean that we cannot expect an inflection point with respect to a 2 nd derivative of a potential system. Such an inflection point designating a speed up of acceleration due to DE exists a billion years ago [10]. Also note that the reason for the failure for (5) to be congruent to (1) is due to

$$
\left[\frac{\partial^{2} U}{\partial\left(\eta^{a}\right)^{2}}=T_{a a}+\lambda(x) g_{a a}\right] \neq 0, \text { for } \forall \eta_{*}^{a} \text { choices }
$$

What (6) tells us is that there is an embedding structure for early universe geometry, some of which may take the form of the following diagram.

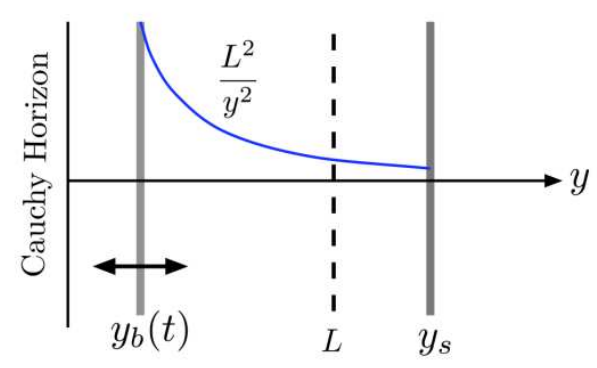

Figure 1, from [3]

\section{Working with a Way to Achieve Energy Injection into the Universe, without Appealing to Fjortoft Theorem for Alleged Instabilities Starting from Padmanabhan Thermodynamic Potential Terms}

Padmanabhan [2] introduced the following discussion as to entropy, namely starting with energy, we have

$$
E=\frac{1}{2} k_{B} \int d n T_{l o c}
$$

And the $n$ value as in (7) is given by

$$
d n=32 \pi \cdot P_{c d}^{a b} \cdot \varepsilon_{a b} \cdot \mathcal{E}^{c d} \cdot d A
$$

Where $P_{c d}^{a b}$ is a so called Lovelock entropy tensor, and $\varepsilon_{a b}$ a bi normal on the co dimension - 2 cross section, and then entropy is stated to be

$$
S \propto \int_{\partial v} d n \propto \int_{\partial v} 32 \pi \cdot P_{c d}^{a b} \cdot \varepsilon_{a b} \cdot \mathcal{E}^{c d} \sqrt{\sigma} d^{D-2} x
$$

The end result, is that energy is induced via the temperature $T_{l o c}$, while [5]

$$
T_{l o c}=N \frac{a^{\mu} n_{\mu}}{2 \pi}=
$$

local acceleration temperature $(10)$

Also, the change in $\mathrm{n}$ can be given by, if $l_{P}$ is the Planck's length value[5]

$$
\Delta n=\sqrt{\sigma} d^{2} x / l_{P}
$$

Looking at (9) and (11) we state that the change in number count given in (11) is really a holographic surface pheonmena, with $\mathrm{N}$ defined [5] 


$$
N=E /\left[(1 / 2) k_{B} T\right]
$$

The upshot is that we can, as implied by $\mathrm{Ng}[11$ ] easily reference a change in entropy via[11],[12],[13]

$$
S \sim n
$$

While having a change in $\mathrm{n}$ as due to a change in the spatial surface of spacetime as given in (11), we have to realistically infer that the local acceleration temperature (10) is from another pre universe contruction and that local instability is ruled out by (5) and (6). This leads us to ask as to what would be an acceptable way to form the formation of mass, i.e. say the mass of a graviton, via external factors introduced into our universe prior to the Electroweak era, in cosmology. To do that, look at if there are two branes on the $A d S_{5}$

space-time so that with one moving and one stationary, we can look at Figure 1 as background as to introduce such external factors in our present space-time universe during its initial expansion phase

\section{Fall out from Adopting Figure 1 and that due to No Instability in the Padamanabhan Supplied Potentials. I.E. a Way to Obtain Graviton Mass Viaa Root Finding Method}

Using [3], [4] what we find is that there are two branes on the $A d S_{5}$ space-time so that with one moving and one stationary, we can look at figure 1 which is part of the geometry used in the spatial decomposition of the differential operator acting uponthe ${ }^{h}$ Fourier modes of the ${ }^{h_{i j}}$ operator [4] . As given by [4], we have that

$$
\left[\partial_{t}^{2}+k^{2}-\partial_{y}^{2}+\frac{3}{y} \cdot \partial_{y}\right] h_{\bullet}=0
$$

Using [3] (and also [14]) the solution to (14) above takes the form of having

$$
h_{\bullet}=H_{i j}=e_{i j} \cdot \exp [i \cdot \omega \cdot t] \cdot(m \cdot y)^{2} \cdot A \cdot J_{2}(m \cdot y)
$$

$e_{i j}$ is a polarization tensor, and the function $J_{2}(m y)$ is a 2nd order Bessel function [14] . A generalization offered by Durrer et al. [3], [4] leads to

$$
h=\left\{\exp [i \cdot \omega \cdot t] \cdot(m \cdot y)^{2} \cdot A \cdot J_{2}(m \cdot y)\right\} \cdot\left(1+\frac{\pi}{4} \cdot(m \cdot \ell)^{2}\right)
$$

With the factor of $\left(1+\frac{\pi}{4} \cdot(m \cdot \ell)^{2}\right)$ coming in due to a boundary condition upon the wall of a brane put in, i.e. looking at [4]. With the right hand side of (16) due to a domain wall tension of a brane

$$
-2 \cdot \partial_{y} H_{i j}=\kappa_{5} \cdot \pi_{i j}^{(T)} \rightarrow 0
$$

This will be in our example set as not equal to zero, in the right hand side, but equal to an extremely small parameter, namely

$$
\left.\partial_{y} H_{i j}\right|_{y=y b}=\kappa_{5} \cdot \pi_{i j}^{(T)} \sim \xi^{+}
$$

With this turned into

$$
\left.\partial_{y} h\right|_{y=y b} \sim \delta^{+}
$$

The right hand side of (19) represents very small brane tension, which is understandable. Then using [3],[4],[14], i.e.

$\left.\partial_{y} h\right|_{y=b b}=\partial_{y}\left\{\exp [i \omega t] \cdot(m y)^{2} \cdot A \cdot J_{2}(m y)\right\} \cdot\left(1+\frac{\pi}{4} \cdot(m \cdot \ell)^{2}\right)_{y=b \infty} \sim \delta^{+}$

and

$$
J_{2}(m y)=\frac{(m y)^{2}}{2^{2} \cdot 2 !} \cdot\left(1-\frac{(m y)^{2}}{2^{2} \cdot 3}+\frac{(m y)^{4}}{2^{4} \cdot 2 ! 3 \cdot 4}-\frac{(m y)^{6}}{2^{6} \cdot 4 ! 3 \cdot 4 \cdot 5}+\ldots\right)
$$

The upshot is, that afterwards,

$$
\begin{aligned}
& \frac{(m y)^{4}}{2^{2} \cdot 2 !} \cdot \frac{1}{y}\left[\begin{array}{l}
\left(1-\frac{(m y)^{2}}{2^{2} \cdot 3}+\frac{(m y)^{4}}{2^{4} \cdot 2 ! \cdot 3 \cdot 4}-\frac{(m y)^{6}}{2^{6} \cdot 4 ! \cdot 3 \cdot 4 \cdot 5}+\ldots\right) \\
-\left(\frac{2 \cdot(m y)^{2}}{2^{2} \cdot 3}+\frac{4 \cdot(m y)^{4}}{2^{4} \cdot 2 ! \cdot 3 \cdot 4}-\frac{6 \cdot(m y)^{6}}{2^{6} \cdot 4 ! 3 \cdot 4 \cdot 5}+\ldots\right)
\end{array}\right] \\
& =\frac{\delta^{+} \cdot \exp [\mp i \omega t]}{A} \cdot\left[1-\frac{\pi}{4} \cdot(m \cdot \ell)^{2}\right]
\end{aligned}
$$

Should the term

$$
\frac{\delta^{+} \cdot \exp [\mp i \omega t]}{A} \cdot\left[1-\frac{\pi}{4} \cdot(m \cdot \ell)^{2}\right] \underset{\delta^{+} \rightarrow 0}{\longrightarrow} 0
$$

Then, (22) is acting much as in [3], and [4], whereas, one is recovering a simple numerical exercise as to obtain a suitable solution as given by (18), and (19) due to [3] where the domain tension of the brane vanishes. The novelty as to this approach given in (22) is to obtain a time dependent behavior of the mass of the graviton,

$$
(m y)=f(t) \Leftrightarrow m \equiv \frac{f(t)}{y}
$$

Needless to say, (22) can only be solved for, numerically, i.e. fourth order polynomial solutions for quartic equations still give over simplified dynamics, especially if (24) holds, and makes things more complicated. This is all being done to keep fidelity with respect to [3], and [4] as a possible feature of brane world dynamics as reflected in [3],[4], as well as certain issues brought up in [15], [16] as to what is a semi classical argument can obtain a usually quantum 
result. We shall now apply it to Mach's principle.

\section{Once a Mechanism for Massive Grasvitons Is Described, Use of Mach's Principle to Link EW Era, with Results of Today, and Why}

We used the following in [17] in order to make a linkage between the early universe, which had a huge vacuum energy, with present day conditions. This makes the primary case which is of the uniformity of physical law, in spite of huge variations as to the DE and Vacuum energy,

Table 1 Time IntervalDynamical consequencesDoes $Q M / W d W$ apply

\begin{tabular}{|c|c|c|}
\hline $\begin{array}{l}\text { Just before Electro- } \\
\text { weak era }\end{array}$ & $\begin{array}{l}\text { Form } \hbar \text { from early E \& M fields, and use Maxwell's Equations with necessary to implement boun- } \\
\text { dary conditions created from change from Octonionic geometry to flat space }\end{array}$ & NO \\
\hline Electro-Weak Era & $\hbar$ kept constant due to Machian relations & $\begin{array}{l}\text { YES } \\
\text { Use (25)as linkage }\end{array}$ \\
\hline $\begin{array}{l}\text { Post Electro-Weak Era } \\
\text { to today }\end{array}$ & $\hbar$ kept constant due to Machian relations & $\begin{array}{l}\text { YES } \\
\text { Wave function of } \\
\text { Universe }\end{array}$ \\
\hline
\end{tabular}

In so may words, the formation period for $\hbar$ is our pre quantum regime. This is incidentally the boundary region before the break down of Octonionic gravity, to our present cosmology. When we get to the present era, and the breakdown of Octonionic geometry, exemplified by spatial commutation relations equaling zero, is when QM applies. Before that regime, QM does not apply.

\section{Getting the Template as to Keeping Information Content Available for Planck's Constant Uniform}

The Machian hypothesis [17] [18], [19] [20] is a way to address a serious issue. The issue is how to keep the consistency of physical law intact, in cosmological evolution. So far, using the template of gravitons and their superpartners, gravitinos, as information carriers, the author has provided a way to argue that Planck's constant remains invariant as from the EW(electroweak era) to the present era. As one can deduce from physical evolution of the cosmos, time variance ofPlanck's constant andtime variation of the fine structure constant would lead to dramatically different cosmological events than what is deduced by obervational astronomy. What we are arguing, using Mach's principle is

Physical law remains invariant in cosmological evolution due to the constant nature/ magnitude of $\mathrm{h}$ bar, the fine structure constant, and $\mathrm{G}$ itself. Volovik indicates the robustness of $\mathrm{G}$ [1] too.

The linkage in information from a prior to the present universe can be thought of as far as the constancy of (19) concerning gravitinos. While we are aware that gravitinos have a short life time, we argue that (19) would have significant continuity at/before the big bang, and also that this is a way of answering the memory question as to how much cosmological memory is preserved from a prior to the present universe structures. Needless to say though there is a complete breakdown in causality before the formation of the gravitinos which is incidentally the pre quantum regime of space-time, i.e. where Octonionic geometry predomi- nates as given by [21] and [22].

The main task the author sees is in experimental verification of the following identity. See (25) below

The motivation of using two types of Mach's principle, one for the Gravitinos in the electroweak era, and then the 2nd modern day Mach's principle, as organized by the author are as seen in (25) as re stated below [17]

$$
\frac{\left.G M_{\text {electro-weak }}\right|_{\text {Super-partner }}}{R_{\text {electro-weak }} c^{2}} \approx \frac{\left.G M_{\text {today }}\right|_{\text {Not-Super-Partner }}}{R_{0} c^{2}}
$$

Once making the double Mach's principle with (25) equal to a constant is done, with $\mathrm{M}=\mathrm{N}$ times $\mathrm{m}$, where $\mathrm{N}$ is the number of a particular particle species, and $\mathrm{m}$ is the net mass of the particle species, then an embedding of quantum mechanics using Mach's principle as part of an embedding space can be ventured upon and investigated experimentally.. It also would allow for eventually understanding if entropy can also be stated in terms of gravitons alone in early universe models as was proposed by Kiefer \& Starobinsky, et. al. [23]. Finally, it would address if QM is embedded in a larger deterministic theory as advocated by t' Hooft [24], as well as degrees of freedom in early universe cosmology as brought up by Beckwith in Dice 2010 [25]. We argue that making this step is consistant with keeping the value of Planck's constant uniform in spite of Avessans theories suggesting it vary in time [26]. To do this, we make extensive use of [27] and [28].

It is now then time to do a re cap and to organize how such speculation can be vetted using experimental proceedures. To do this we re cap what can be said about traces massive gravitons can be detected, prior to our conclusion

\section{Semiclassical Method of Obtaining Graviton Mass Procedure Cannot Be Ruled out, and It Impacts Relic GW Searches}

First of all, review the details of a massive graviton im- 
print upon $h_{i j}$, and then we will review the linkage between that and certain limits upon $h_{\bullet}$ As read from Hinterbichler [29], if $r=\sqrt{x_{i} x_{i}}$, and we look at a mass induced $h_{i j}$ suppression factor put in of $\exp (-m \cdot r)$, then if

$$
\begin{gathered}
h_{00}(x)=\frac{2 M}{3 M_{\text {Planck }}} \cdot \frac{\exp (-m \cdot r)}{4 \pi \cdot r} \\
h_{0 i}(x)=0 \\
h_{i j}(x)=\left[\frac{M}{3 M_{\text {Planck }}} \cdot \frac{\exp (-m \cdot r)}{4 \pi \cdot r}\right] . \\
\left(\frac{1+m \cdot r+m^{2} \cdot r^{2}}{m^{2} \cdot r^{2}} \cdot \delta_{i j}-\left[\frac{3+3 m \cdot r+m^{2} \cdot r^{2}}{m^{2} \cdot r^{4}}\right] \cdot x_{i} \cdot x_{j}\right)
\end{gathered}
$$

Here, we have that these $h_{i j}$ values are solutions to the following equation, as given by [29], [30], with D a dimensions value put in.

$$
\left(\partial^{2}-m^{2}\right) h_{\mu v}=-\kappa \cdot\left[T_{u v}-\frac{1}{D-1} \cdot\left(\eta_{u v}-\frac{\partial_{\mu} \partial_{v}}{m^{2}}\right) \cdot T\right]
$$

To understand the import of the above equations, set

$$
\begin{aligned}
& M=10^{50} \cdot 10^{-27} \mathrm{~g} \equiv 10^{23} \mathrm{~g} \propto 10^{61}-10^{62} \mathrm{eV} \\
& M_{\text {Plank }}=1.22 \times 10^{28} \mathrm{eV}
\end{aligned}
$$

We should use the $m_{\text {massive-graviton }} \sim 10^{-26} \mathrm{eV}$ value in (29) above.

In reviewing what was said about (27),(28) we should keep in mind the overall Fourier decomposition linkage between $h_{\bullet}, h_{i j}$ which is written up as

$$
h_{i j}(t, x ; k)=\frac{1}{(2 \pi)^{3 / 2}} \int d^{3} k \sum_{\bullet=+, \otimes} e^{i k \cdot x} e_{i j}^{\cdot} h_{\bullet}(t, y ; k)
$$

The bottom line is that the simple de composition with a basis in two polarization states, of,$+ \otimes$ will have to be amended and adjusted, if one is looking at massive graviton states.

In addition further developments as to (31) could influence giving a semi classica interpretation as to entrophic origins of gravity, along the lines brought up by both t'Hooft, indirectly [24], andLee [31] directly.

\section{Conclusion}

What has been presented here is a unified attempt to link massive gravitons, with the existence of a uniform Planck's value, and also the issues presented in [1] and [2]. "Fjortoft theorem" not applying is a wake up call for cosmologists to reconcile the fact that thermodynamic potentials of space- time as given by [5] in by themselves do not allow for instabilities, and that other mechanisms, including judicious applications of Machs principle, from early to present space-time may be necessary to understand cosmological evolution. It is striking that according to [2] that as Einstein insightfully ascertained in 1918 that the cosmological parameter called the "cosmological constant" being either zero or almost zero would imply or be linked to a highly non homogeous matter-energy distribution of space-time but that the early universe, as far as what can be ascertained by WMAP and other probes was highly homogeneous. The disparity in what is predicteded by [2] is even more striking when one realizes that to answer Volovik's [1]'s statement about the non applicability of small Casmir plates for generating non zero cosmological vacuum energy, due to their finite size, would be to go to giant [3] and [4]infinite sized Casmirplates using [8] and [9] infinite sized brane and anti branes. If Volovik's hypothesis as to DE being the source of vacuum energy is correct, and [3] and [4] can be linked to massive gravitons as attempted to be shown here, then by [6] there is a ready explanation as to how massive gravitons as created by giant Casmir plates [3], [4] could be relevant today. We also state, that judicious tweaking of [3] and [4] plus applications of Mach's principle [17],[18],[19],[20] could be a further extension of the Higgs boson mass formation mechanism, which is partly verified by CERN but is certainly not the final story. This work is meant to complimment Higgs Boson formation of mass with another view point, not replace it. Keep in mind that the author views Quantum processes as embedded in a larger deterministic theory much as t'Hooft does in [24] and that appealing to a non quantum mechanical creation of Planck's constant as done in [27], and [28] is no accident. The author finally views Mach's principle as giving a uniform value for Planck's constant in opposition to [26] as essential as keeping uniform physical laws during cosmological evolution since the alternative is frankly a recipede for undefinabilty of physics as we know it. That uniform Plansk's constant, plus Mach's principle is a way to make the Brane-anti Brane casmir derivation of DE, as in reference to re accelleration [6] viable and to give reality to [25]. The remaining challenge is experimental, in verification which is given by [29],[30], and [31]. To which the author says, good luck, we will all need it in this endeavor as seen by the work in [31].

\section{Acknowledgements}

This work is supported in part by National Nature Science Foundation of China grant No110752

\section{References}

[1] G.E. Volovik, "Vacuum Energy: Myths and Realities", arXIV: gr-qc/0604062 v2, April 16, 2006

[2] A. Einstein,Principielles Zur Allegmeinen Relativistatstheori, 
Allen Der Physik,55,241-244(1918)

[3] R. Durrer et.al., "Dynamical Casmir effect for gravitonsin Bouncing Braneworlds",2009, http://theory.physics.unige.ch/ durrer/papers/casimir.pdf; Earlier version http://www.scribd.com/doc/79001898/RuthDurrer-and-Marcus-Ruser-The-dynamical-Casimir-effect-inbraneworlds ; Version put into PRD. Marcus Ruser, Ruth Durrer, "Dynamical Casimir effect for gravitons in bouncing braneworlds", Phys.Rev.D76:104014,2007

[4] R. Durrer, "Branewords", http://arxiv.org/pdf/hepth/0507006v1.pdf

[5] Thanu Padmanabhan 2011 J. Phys.: Conf. Ser. 306 01200;"Lessons from Classical Gravity about the Quantum structure of space-time", DICE 2010, editor T. Elze, http://iopscience.iop.org/1742-6596/306/1/012001

[6] A. Beckwith, "Identifying a Kaluza Klein Treatment of a Graviton Permitting a deceleration parameter Q $(Z)$ as an Alternative to Standard DE", Journal of Cosmology, 2011, Vol.

http://journalofcosmology.com/BeckwithGraviton.pdf

[7] J. Pringle and A. King; Astrophysical Flows, Cambridge University Press, New York, 2007

[8] J. Koury, P.J. Steinhardt,N. Turkok, PRL92, 031302(2004),hep-th/0307132

[9] J. Koury, P.J. Steinhardt,N. Turkok, PRL91, 161301(2003), astro-ph/0302012

[10] A. Beckwith, "Gravitons, Gravitinos, and Using Mach's Principle to Make a Statement of Equivalent Information in a Gravitino-Graviton Correspondence of Critical Information Exchange from Electroweak to Present Era", http://vixra.org/abs/1204.0089

[11] Y.J. Ng, Entropy 10(4), pp441-461 (2008); Y.J. Ng and H. van Dam, FoundPhys30, pp795-805 (2000);

[12] Y.J. Ng and H. van Dam, Phys. Lett. B477, pp429-435 (2000);

[13] Y.J. Ng, "Quantum Foam and Dark Energy", in the conference International work shop on the Dark Side of the Universe,

http://ctp.bue.edu.eg/workshops/Talks/Monday/QuntumFoa mAndDarkEnergy.pdf

[14] Bessel functions and Hankel Functions: NIST: http://dlmf.nist.gov/10.2

[15] C.F. Monnin, and G.M. Prok, "Comparison of Gryzinski's and Born's Approximation for Inelastic scattering in Atomic Hydrogen",

http://ntrs.nasa.gov/archive/nasa/casi.ntrs.nasa.gov/1965001 7671_1965017671.pdf;

[16] M. Gryzinski, "Classical theory of Electronic and Ionic Inelastic Collisions”Phys Rev Vol. 115, no2, July 15, 1959, pp 374-383

[17] A.W. Beckwith, "Is Quantum Mechanics Involved at the Start of Cosmological Evolution? Does a Machian Relationship Between Gravitons and Gravitinos Answer This Question?", http://vixra.org/abs/1206.0023

[18] S. Mishra, J. Christian, "Effective Theory of Quantum Gravity: Soluble Sector", Abramis Academic Publishing, (March 4, 2011);

[19] S. Mishra, "The Quantum theory of the Big Bang: Effective theory of Quantum Gravity", pp 205-228, From 'The Big Bang, Theory, Assumptions and Problems' Ed.J.O.'Connell, A.Hale, Nova Book publishers, 2012, New York, New York;

[20] J. Christian, "Exactly solvable sector of Quantum Gravity", ArXIV gr-qc 9701013 version 4, November 7, 2005 \& PRD 56, No8, 15 Oct1997, pp 4844-4877

[21] L. Crowell, "Quantum Fluctuations of Space Time", World Scientific Series in Contemporary Chemical Physics, Vol25, World Scientific, PTE, LTD, 2005, Singapore

[22] A. Beckwith, "Octonionic Gravity Formation, Its Connections to Micro Physics," Open Journal of Microphysics, Vol1 No1, 2011, pp13-18

[23] C. Kiefer, D. Polarski, A.A Starobinsky, "Entropy of gravitons produced in the early Universe", PRD 62, 043518 (2000) [6 pages]; arxiv gr-qc/9910065

[24] G. t' Hooft, "Quantum Mechanics as a dissipative deterministic system" http://arxiv.org/PS_cache/grqc/pdf/9903/9903084v3.pdf

[25] A. Beckwith, "How to use the cosmological Schwinger principle for energy flux, entropy, and "atoms of spacetime" to create a thermodynamic space-time and multiverse", http://iopscience.iop.org/1742-6596/306/1/012064, in"5th International Workshop DICE2010: Space-TimeMatter - Current Issues in Quantum Mechanics and Beyond", T. Elze ed. http://iopscience.iop.org/17426596/306/1;jsessionid=A05372A78C18D970BF35F40A9A $863 \mathrm{~B} 51 . \mathrm{c} 2$

[26] A.K. Avessian, "Planck's constant evolution as a cosmological evolution test for the early universe", .Gravitation and Cosmology, 2009, Vol. 15, number 1, pp 10-12

[27] U. Bruchholz; "Derivation of Planck's constant from Maxwell's Electrodynamics", Progress in Physics, V4, October 2009, page 67; http://www.pteponline.com/index_files/2009/PP-19-07.PDF

[28] U. Bruzchholz, "Key notes on a geometric theory of fields", Progress in Physics, 2009, v.2, 107-113.

[29] K.Hinterbichler, "Theoretical Aspects of Massive Gravity", http://arxiv.org/abs/1105.3735.pdf

[30] http://web.mit.edu/redingtn/www/netadv/Xgravitati.html

[31] Jae-Weon Lee, "On the Origin of Entropic Gravity and Inertia”, http://arxiv.org/abs/1003.4464 